\title{
Proceso de adaptacion del inventario de la relación de ayuda de G.T. Barrett-Lennard ${ }^{1}$.
}

\author{
Alejandro Celis $\mathrm{H}^{2}$
}

\section{Resumen}

La presente investigación expone la realización de las etapas iniciales de la adaptación al español del Inventario de la Relación de Ayuda de G.T. Barrett-Lennard, en su versión OS-M-64. Se exponen diversas consideraciones respecto a la importancia del punto de vista del "receptor" de la relación, respecto a la utilidad del cuestionario y respecto a la metodología empleada en su adaptación. Se discuten dificultades metodologicas halladas en la investigación y se entrega una versión completa del Inventario y de la forma de utilizarlo.

Palabras Clave: Barrett-Lennard, Inventario de la Relación, Cambio terapéutico, percepción del cliente.

\section{Abstract}

This paper presents the initial steps of an adaptation to spanish of the OS-M-64 version of G.T. BarrettLennard's Relationship Inventory. Also discussed in the article are the importance of the relationship's "receptor" point of view, the usefulness of the questionnaire and different aspects and difficulties related to the methodology used in its adaptation. Also included are a complete version of the Inventory and instructions for its use.

Key Words: Barrett-Lennard, Relationship Inventory, therapeutic change, client's perception.

\section{Introducción}

La importancia de la calidad de la relación interpersonal para detonar cambio en las personas ha sido extensamente investigada y verificada por diversos autores, especialmente Carl Rogers (1957, 1967). El tipo de relación que establece un terapeuta, un profesor, una madre o un padre o un simple amigo o compañero de trabajo puede ser un factor que facilite el que en la persona se establezca un modo de funcionamiento sano. Esto ha sido más intensamente investigado en el caso del hijo con respecto a los padres (Gordon, T. 1970), en el del cliente con su terapeuta (Rogers, C. 1967) el del alumno con su profesor (D’Augelliy Weener, 1977) y el del subordinado hacia su lider (Gordon, T., 1980).

En 1957, Rogers definió -estableciendo lo que actualmente es considerado un hito paradigmático en la psicoterapia- los elementos claves en la calidad de la relación terapéutica. Estas condiciones eran:(1)Que haya dos personas en contacto psicológi-

\footnotetext{
${ }^{1}$ La presente investigación se dio en el marco del Concurso de Proyectos de Investigación Internos (Intradisciplinaria -Psicología- $\left.\mathrm{N}^{\mathbf{0}} 2024\right)$ de 1996 de la Universidad Diego Portales. Inicialmente, contó con el apoyo metodológico de la Srita. Paulina Cuevas V., la que se retiró de la misma antes de su término.

${ }^{2}$ Psicólogo Clínico Transpersonal titulado en la Universidad de Chile en 1977. Docente de las Escuelas de Psicología de la U. Diego Portales desde 1987, y de la Universidad Central desde 1997. Supervisor Acreditado por la Comisión Nacional de Acreditación de Psicólogos Clínicos (1995). E-mail: savanna@entelchile.net
} 
co; (2)Que la primera-llamada "cliente"-se encuentre en estadode incongruencia, siendo vulnerableoestando angustiada;(3)Quelasegunda persona, llamada "terapeuta" sea congruente ointegrada en la relación (4)Que elterapeuta sientauna consideración positivaincondicional hacia el cliente (5)Que el terapeuta experimente una comprensión empática del marco de referenciainterno del cliente y trate de comunicarle su experiencia, y (6) Que la comunicación al cliente de la comprensión empática y la consideración positiva incondicionaldel terapeutase logre en ungradoalmenos mínimo(Rogers, C., 1957).

Resulta, entonces, valioso e interesante -a lo que apunta la sexta condición estipulada- evaluar la percepción del cliente/hijo/alumno/subordinado del grado en que dichos elementos se hallan presentes en la relación que le brindan. Con esto en mente, G.T. Barrett-Lennard desarrolló (1962), en base a las condiciones terapéuticas descritas por Rogers, un Inventario de la Relación. Este Cuestionario es respondido por el "receptor" o cliente de la relación terapéutica, con respecto a las condiciones brindadas por el "emisor" o terapeuta. El Inventario ha sido estudiado continuamente desde entonces (Barrett-Lennard, G.T., 1997a), y este tipo de enfoque coincide con las tendencias actuales en la investigación del proceso terapéutico: Según algunos autores, la contribución del paciente a la relación es más poderosa que la del terapeuta. En particular, la vivencia del paciente y su compromiso con el proceso terapéutico constituyen elementos de gran importancia (Winkler etal, 1993).

Inicialmente, Barrett-Lennard destinó la prueba a ser utilizada especialmente en la relación terapeuta-cliente; sin embargo, ya desde muy temprano se realizaron estudios en relaciones de otro tipo. El mismo Barrett-Lennard, en un estudio de confiabilidad test-retest de la prueba, solicitó a los sujetos que la respondiesen respecto a una relación cercana y de larga duración (Barrett-Lennard, G.T., 1962). En el mismo estudio, se cita una investigación preliminar de Berlin (1960) en que solicitó a un grupo de estudiantes de sexo femenino que evaluasen su relación con compañeras de curso.

Diversos estudios y revisiones posteriores de la prueba arrojaron conclusiones favorables respecto a su validez y confiabilidad (Litwack, Getson y Saltzman, 1968). Una versión inicial de la prueba en español fue asimismo utilizada en nuestro país (Didier, M. 1975); sin embargo, ésta no fue convenientemente adaptada para este fin ${ }^{3}$.

\section{La Investigación Original}

El estudio original fue un intento preliminar de conectar causa y efecto en el proceso terapéutico, teniendo como base teórica la concepción de Carl Rogers de las "condiciones necesarias y suficientes" del cambio terapéutico (Rogers, C.R., 1957). La investigación se inició tomando como base la idea de que el cambio terapéutico de la personalidad ocurre en proporción al grado en que el cliente percibe ciertas cualidades en la respuesta del terapeuta hacia él o ella. Las cualidades mostradas por el terapeuta que el Inventario consideró originalmente fueron: su grado de consideración positiva, el grado de la incondicionalidad de su aprecio, su comprensión empática, sucongruencia o autenticidad ysu disposición a ser conocido por sucliente.

En la versión original, el Inventario constaba de 92 items, los que se hallaban subdivididos en 5 Escalas: Aprecio, Comprensión Empática, Congruencia, Incondicionalidad del Aprecio y Disposición a ser Conocido. La Escala Disposición a ser Conocido fue posteriormente eliminada, por diversas consideraciones de tipo metodológico. Así, la versión (OS-M-64) del Inventario que fue adaptada en esta investigación consta sólo de 64 items.

\section{LasEscalas}

\section{(1) COMPRENSION EMPATICA.- (E)}

La Comprensión Empática es concebida como el grado en el cual una persona es consciente de lo que en este momento le ocurre a otra persona internamente. Cualitativamente, es un intento activo por conocer enteramente lo que vivencia en este momento otra persona, un esfuerzo por captar su co- 
municación y el significado de ésta, y por traducir sus palabrasy señales en significados experienciales que coincidan al menos con aquellos aspectos que le son más importantes en este momento. Es un percibir lo que vivencia el otro "detrás" de lo que comunica explicitamente, pero teniendo presente que este vivenciar se origina y procede del otro. Es quizás ésta la cualidad de la relación que ha sido más extensamente investigada, tanto en el país -en numerosas Tesis de Grado dirigidas por el Prof. Gabriel Reyes en la Universidad de Chile y Universidad Central-, como en el extranjero (BarrettLennard, G.T., 1981, 1988, 1993, 1997b; Bohart, Ay Greenberg, L., 1997; Book, H.E., 1988; Cohen, J., 1994), a pesar de que el autor K.B. Clark (1980) señala que esta variable no ha recibido suficiente atención. De uno u otro modo, la puntuación de la escala indica el grado en que el cliente se siente comprendido por su terapeuta, en el sentido de que éste capte con la mayor exactitud posible lo que sienta o piense el primero.

\section{(2) NIVEL DE APRECIO.- (Ap)}

El Aprecio es aquí definido como el componente afectivo de la respuesta de una persona hacia otra. Esta respuesta puede incluír diversas cualidades e intensidades de sentimientos "positivos" (como respeto, simpatía, aprecio, etc) y "negativos" (como desagrado, impaciencia, desprecio, etc). El Nivel de Aprecio es la tendencia general (en un periodo determinado de tiempo) hacia donde apuntan las diversas reacciones afectivas de una persona en relación a otra. El extremo inferior de esta dimensión representa la predominancia e intensidad máximas de los sentimientos de índole negativa, no sólo la ausencia de sentimientos positivos.

\section{(3) AUTENTICIDAD O CONGRUENCIA.- (C)}

La Congruencia que exhibe una persona en una relación determinada con otra persona es el grado en el cual la primera se halla funcionalmente integrada en el contexto de su relación con la segunda, de modo que hay ausencia de conflicto o inconsis- tencia entre su experiencia total, su consciencia y su comunicación explicita. El concepto se centra, a nivel teórico, en la consistencia entre la vivencia total y el darse cuenta de la persona, lo que se considera como el ingrediente principal de la congruencia entre consciencia y comunicación. La puntuación de la Escala indica, entonces, el grado en que el cliente percibe que su terapeuta se muestra tal como es, sin ocultarse tras máscaras o roles, y el grado de disposición que muestra en cuanto a que su cliente conozca sus sentimientos y forma de ser.

\section{(4) INCONDICIONALIDAD DEL APRECIO.-} (IA)

Este concepto se relaciona especificamente con el grado de variabilidad que exista en la respuesta afectiva de una persona con respecto a otra. La Escala mide el grado en el que el aprecio brindado por el terapeuta (sea cual sea el grado de este aprecio) está sujeto a determinadas condiciones: actitudes o conductas deseadas. En su polo positivo, esta dimensión implica que todas las experiencias del cliente afectan al terapeuta como igualmente dignas de consideración positiva, sin distinguir ninguna como más o menos digna. En su polo negativo, el terapeuta impone al cliente -conscientemente o no- su propia escala de valores, considerando dignas de aprecio algunas vivencias del cliente $\mathrm{e}$ indignas de aprecio, otras.

Una investigación ya mencionada (Winkler et al, 1993) concluye: "En general, el no ser criticado/a parece seruno delos aspectos que más se valora en la relación terapéutica". Una de las conclusiones de este estudio fue, "Porotra parte, en la investigación realizadaqueda corroborada la importancia quetienen las actitudes del terapentapara el cambio psicológico: especialmente, las actitudes deaceptación yempatía. Eneste aspecto, los resultados coincidenconel planteamientode Rogers (1957) y los de Parloffetal.(1978) y de Stiles et al. (1986) respecto de la importancia de la alianza terapéutica. Estas actitudes parecen contribuír a la creación deunclimapsicológicoque daconfianzay seguridadal paciente". 


\section{Procedimiento Metodológico}

El propósito de la presente investigación fue adaptar adecuadamente una versión en español del Inventario para su utilización en la investigación y evaluación de la relación brindada por alguno de los agentes ya mencionados. La metodología utilizada se enfrentó a una serie de trastornos e inconvenientes que resultaría demasiado extenso detallar. Uno de los principales obstáculos fue el verificar que, a pesar de que la estadística es presentada como ciencia "dura", existen en ella multiplicidad de criterios. Por ejemplo, lo que para un experto es una Estandarización, para otro apenas alcanza a ser una Adaptación ${ }^{4}$.

Se siguió el procedimiento de adaptación resenado para el Inventario de Actualización Personal (Celis, A. et al, 1976). Expongo a continuación los pasos en que se avanzó en su adaptación al medio chileno.

(a) traducción inglésespañol del Inventario por un traductor experto y con conocimientos del lenguaje psicológico: ésta fue realizada por el autor de este artículo, dada su extensa experiencia como traductor inglés-español;

(b) reproducción de la traducción y envio a 7 jueces expertos en ambos idiomas, los que actuaron por separadoy dispusieron tanto de laversión en inglés como de su traducción. Los juecesfueron: una secretaria trilingüe de una Embajada Europea; dos secretarias bilingües de un organismo de Naciones Unidas; un alto funcionario del Banco Mundial; una traductora experta inglés-español de la Editorial chilena Cuatro Vientos, y dos secretarias de nacionalidad norteamericana con fluído dominio del idioma español.

(c) recepción de criticas y observaciones de estosjueces: confecciónde una segundaversióndel Inventario y realización de una aplicación piloto. Las sugerencias que realizaron fueron recogidas y evaluadas por tres psicólogos bilingües en términos de la frecuencia con que se realizaban (por ejemplo, si una frase era cuestionada más de una vez) y la atingencia de éstas dentro del lenguaje psicológico en uso (por ejemplo, si la sugerencia -aun cuando fuese la única respecto a esa frase particularparecía ser un aporte real a la claridad e inteligibilidad de la frase).

Así recogidas e integradas las criticas y observaciones de los jueces, se confeccionó una segunda versión del Inventario, la que fue sometida a una aplicación piloto con 60 alumnos de Enseñanza Media, cuya edad fuese igual o mayor a 17 años (calificando a sus Profesores Jefes) y 30 clientes adultos en terapia (calificando a sus terapeutas), con el propósito de constatar si esta aplicación piloto detectaba dificultades de comprensión en alguno(s) de los item(es). Fue destacable observar que la claridad del lenguaje del Inventario fue tal que no se observaron en la muestra elegida mayores dificultades de comprensión, salvo casos aislados debidos al desconocimiento de palabras de uso común.

El proyecto original establecia que, "una vez realizadas las posibles correcciones, se aplicará el Inventario a una muestra de alrededorde 200 individuos (de ambossexos, deedadesentre $17 y 45$ añosquesehayan sometidoaunapsicoterapia)conel fin de estableceruna modalidaddecorrección(oategonías de puntajes)queconsidere las características del universo poblacional chileno". Sin embargo -con la intención de tomar en cuenta las observaciones realizadas por el evaluador externo en el Concurso de Proyectos- nos propusimos un esquema de trabajo que reemplazaba lo anterior por pasos que implicaban evaluar a 50 clientes en dos momentos diferentes -la primera ocasión, entre la segunda y la tercera sesión; y la segunda, en la octava sesión o antes si el término de la terapia ocurria con anterioridad- de su terapia con psicoterapeutas de diversas orientaciones.

El procedimiento implementado fue preparar, para cada paciente que fuese posible sujeto de la muestra, un sobre sellado que contenía una carta de presentación del Proyecto y una invitación a participar en él, un ejemplar del Inventario, una hoja de respuesta y un sobre con estampilla dirigido a los investigadores, dentro del cual el paciente debía hacernos llegar su hoja de respuesta contestada. El sobre sellado que contenia todas estos docu- 
mentos debía ser, idealmente, entregado a la o el paciente por la secretaria de la Consulta o Consultorio -con el acuerdo tácito del psicoterapeuta-, de modo que el paciente sintiese la mayor libertad posible para decidir si participaba o no en el Proyecto; $y$, en caso de decidir hacerlo, que sintiese la mayor libertad para responder honestamente.

Estos pasos, que metodológicamente parecen obviamente convenientes y fáciles de implementar, se encontraron en la práctica con obstáculos insospechados, a saber:

(b) una vez establecida una muestra de psicoterapeutas dispuestos a ser evaluados por sus pacientes, les fueron entregados los sobres sellados respectivos, quedando a la espera los investigadores de recibir por correo las respuestas de los pacientes. A pesar de la colaboración de los terapeutas participantes, más de dos meses después sólo se habia recibido un total de tres cartas, correspondientes a la primera evaluación de tres pacientes diferentes.

(c) escasa cooperación de los centros de atención contactados (dos consultorios de Universidades privadas y el de un organismo privado de seguridad laboral);

(d) problemas "teóricos" (de orientación psicoterapéutica) planteados por la Dirección para que los pacientes de un Consultorio de un organismo privado que atiende público fuese sometido a responder el Inventario;

Ante estas dificultades -las que no nos fue posible superar, a pesar de nuestras gestiones respecto a conseguir la colaboración de Centros de Atención en que pudiese asegurarse un sistema más organizado de control de la entrega y devolución de los Cuestionarios- sólo nos fue posible verificar que la investigación no podría ser finalizada del modo propuesto en los plazos propuestos.

\section{Resultados}

Del Cuestionario resultante puede decirse que es una traducción óptima del original. Cada una de las cuatro Escalas del Inventario incluye un total de 16 items: 8 de ellos en un sentido positivo o deseable $(+)$ y 8 en un sentido negativo o indeseable (). La Escala a la cual corresponde cada item y su signo (+) o (-) están indicados en el cuestionario adjunto -para fines de este artículo- mas es deseable eliminar dichos datos del Cuestionario que se entregue para ser respondido.

El puntaje con el que es evaluado cada ítem por la persona que responde tiene una variación posible $\mathrm{de}+3 \mathrm{a}-3$. La forma de corregir se inicia invirtiendo el signo (+) o (-) de la respuesta dada por el sujeto en los items que en el Inventario se indican con signo negativo (-), y luego sumar, aritméticamente, los puntajes de los items de cada Escala. Es asi que, por ejemplo, un puntaje de +3 aumentará el puntaje total, y un puntaje de -3 lo reducirá. El puntaje máximo posible, para cada una de las Escalas, es + 48; y el puntaje mínimo, de - 48. Esto corresponde, respectivamente, a +3 o-3 multiplicado por el total de ítems de cada Escala, que es 16.

En el Inventario original, los puntajes no sufrian ninguna conversión, excepto la de invertir el signo de la respuesta dada por el sujeto en los ítems que se indican inicialmente con signo negativo. El autor proponía basar la conclusión diagnóstica del Inventario en la evaluación del peso obvio de cada puntaje: queda para futuros estudios el definir, de ser necesario, categorias en los puntajes de cada Escala; en el intertanto, los puntajes obtenidos deberán ser evaluados en forma cualitativa. El Inventario se presenta en forma completa como Anexo al final de este artículo.

\section{Referencias Bibliográficas}

Barrett-Lennard, G.T. (1962) Dimensions of therapist response ascausal factors in therapeutic change. Psychological Monographs, 76 (whole $\mathrm{N}^{\circ}$ 562).

Barrett-Lennard, G.T. (1981) The Empathy Cycle: refinement of a nuclearconcept. Joumal of Counseling Psychology, 28, 91-100.

Barrett-Lennard, G.T. (1988) Listening. Person-centered review, vol.3, No 4, Sage Publications.

Barrett-Lennard, G.T. (1993) The phases and focus of empathy. British journal of Medical Psychology, 66, 3-14. 
Barrett-Lennard, G.T. (1997a) Relationship Inventory Resource Bibliography. En preparación, actualizado hasta el 11 de Mayo de 1997).

Barrett-Lennard, G.T. (1997b) The recovery of empathy-toward others and self. En Bohart, Ay Greenberg, L. (1997) (ob. Cit)

Berlin, J . (1960) Some autonomic correlates of therapeutic conditions in interpersonal relationships. Unpublished doctoral dissertation, University of Chicago. Citado en Barrett-Lennard, G.T. (1962) (Ob.cit).

Book, H.E. (1988) Empathy: misconceptions and misuses in psychotherapy. American Journal of Psychiatry, 145:4.

Bohart, Ay Greenberg, L. (1997) Empathy reconsidered. New Directions in Psychotherapy. American Psychological Association, Washington.

Celis, A., Farías, R. elturra, A. (1976) Estandarización del Inventario de Actualización Personal(P.O.I.)en una muestra de estudiantes de la Universidad de Chile. Tesis para optar al título de Psicólogo, U. de Chile.

Clark, K.B. (1980) Empathy: a neglected topic in psychological research. American Psychologist, vol.35, No2, 187-190.

Cohen, J . (1994) Empathy toward Client Perception of Therapist Intent: Evaluating One's Person-Centeredness. Person Centered Journal. Kutztown University, Australia.
D'Augelli, Weener (1977) Manual del Padre. The Pennsylvania State University.

Didier, M. (1975) Las habilidades interpersonales del terapeuta y el cambio en la personalidad del cliente. Tesis para optar al titulo de Psicólogo, U. Católica de Chile.

Gordon, T. (1970) Teoria de relaciones interpersonalessanasy un programade entrenamientode padres. Cap. 21 de Newdirections in Client-centered therapy, de J.T. Harty T.M. Tomlinson. HoughtonMifflin.

Gordon, T. (1980) L.E.T.: lideres eficaz y técnicamente preparados. Diana, México.

Hoffman, M.L. (1977) Sex differences in empathy and related behaviors. Psychological Bulletin, vol. 84, No4, 712-722.

Litwack etal. (1968) Research in Counseling. F.E. Peacock, Illinois.

Rogers, Carl R. (1957) The necessary and sufficient conditions of therapeutic personality change. J. of Consulting Psychology, 21.

Thomas, A. y Ubilla, M. (1992) Adaptación y obtención de normas provisorias del Inventario de Autoestima de Coopersmith (SEI-forma A) parajóvenes estudiantes de 7ºbásico a 4 ${ }^{\circ}$ medio del Gran Santiago. Tesis para optar al título de Psicólogo, U. Diego Portales, Santiago de Chile.

Winkler, M.I. et al. (1993) El cambio psicológico desde la perspectiva de los consultantes. Terapia Psicológica $\mathrm{N}^{\circ} 20$, Santiago de Chile. 


\section{Inventario de la Relación deG.T. Barret-Lennard}

Adaptado de la Forma OS-M-64

por A. Celis (1998)

A continuación hay una lista de formas en que una persona determinada puede sentirse o comportarse en su relación con otra persona.

Considere cada afirmación en referencia a su relación actual con la persona que se le indique.

Indique en la Hoja de Respuestas, frente al número correspondiente, cuán fuertemente siente usted que cada afirmación es verdadera o falsa en esta relación. Por favor marque todas las afirmaciones. Escriba $+3,+2,+1,-1,-2$ o -3 , que corresponden a las siguientes respuestas:

+3 = Si, tengo la absoluta convicción de que esto es verdad.

$+2=\mathrm{Si}$, siento que esto es verdad.

$+1=\mathrm{Si}$, siento que probablemente esto es verdad; o más verdadero que falso.

$-1=$ No, siento que esto es probablemente falso, o más falso que verdadero.

$-2=$ No, siento que esto no es verdad.

$-3=$ No, tengo la absoluta convicción de que esto no es verdad.

\section{INDIQUE SUS RESPUESTAS EN LA HOJA DERESPUESTAS. \\ NO HAGA MARCAS EN ESTE CUESTIONARIO.}

$\mathrm{Ap}+$ 1.- Me respeta como persona.

$\mathrm{E}+$ 2.- Quiere entender cómo yo veo las cosas.

Ia- 3.- Su interés por mí depende de lo que yo haga o diga.

$\mathrm{C}+$ 4.- Se siente cómodo(a) y a gusto en nuestra relación.

Ap + 5.- El (ella) siente real agrado por mí.
E- $\quad$ 6.- Puede que entienda mis palabras, pero no se da cuenta de lo que yo siento.

Ia + 7.- Que yo esté contento(a) o descontento(a) conmigo mismo(a) no tiene efecto sobre lo que él (ella) siente por mí.

C - 8.- Siento que se pone una máscara o actúa un rol cuando está conmigo.

Ap - 9.- Es impaciente conmigo.

$\mathrm{E}+$ 10.- Casi siempre sabe con exactitud lo que quiero decir.

Ia - 11.- Según mi comportamiento, algunas veces tiene mejor opinión de mí que en otras.

$\mathrm{C}+$ 12.- Siento que es verdadero(a) y auténtico(a) conmigo.

$\mathrm{Ap}+$ 13.- Me siento apreciado(a) por él (ella).

E- 14.- Lo que yo hago, él (ella) lo ve desde su propio punto de vista.

$\mathrm{Ia}+$ 15.- Lo que siente por míno depende de mis sentimientos por él (ella).

C - 16.- Se siente incómodo(a) cuando yo pregunto o hablo respecto a ciertos temas.

Ap - 17.- Le soy indiferente.

$\mathrm{E}+$ 18.- Generalmente intuye o se da cuenta de lo que estoy sintiendo.

Ia - 19.- Quiere que yo sea de una forma determinada.

$\mathrm{C}+$ 20.- Casi siempre siento que lo que él (ella) dice expresa en forma exacta lo que siente $o$ piensa en ese momento.

Ap - 21.- Me encuentra más bien aburrido(a) y poco interesante.

E- 22.- Su actitud frente a ciertas cosas que yo hago o digo le impiden entenderme.

$\mathrm{Ia}+$ 23.- Puedo o podría criticarlo(a) o apreciarlo(a) abiertamente sin que cambie lo que él (ella) siente por mí.

C - 24.- Quiere que yo crea que me entiende ole agrado más de lo que en realidad me entiende o le agrado.

Ap + 25.- Le importo.

E- 26.- A veces piensa que yo siento algo de cierta manera porque así lo siente él (ella). 
Ia - $\quad$ 27.- Hay cosas de mi que le agradan y otras cosas que no le agradan.

$\mathrm{C}+$ 28.- No evita nada que es importante para nuestra relación.

Ap - 29.- Siento que no aprueba mimanera de ser.

$\mathrm{E}+\quad$ 30.- Entiende lo que quiero decir aún cuando a mi me resulte dificil expresarlo.

$\mathrm{Ia}+$ 31.- Su actitud hacia mi es siempre la misma: no se siente satisfecho(a) conmigo en algunas ocasiones y critico(a) 0 desilusionado(a) en otras.

C - 32.- A veces se siente realmente incómodo(a); sin embargo, hacemos como que no nos damos cuenta y seguimos adelante.

Ap - 33.- Se limita a tolerarme.

E + 34.- Generalmente capta la totalidad de lo que quiero decir.

la - $\quad 35 .-$ Si me enojo con él (ella), él (ella) también se enoja o se siente dolido(a).

$\mathrm{C}+$ 36.- Frente a mi, expresa sus verdaderas impresiones y sentimientos.

Ap + 37.- Es amistoso(a) y cálido(a) conmigo.

E- 38.- Simplemente pasa por alto algunas cosas que yo siento o pienso.

Ia + 39.- Cuánto yo le gusto o disgusto no cambia por lo que yo le diga sobre mí mismo(a).

C - 40.- A veces siento que él (ella) no se da cuenta de lo que realmente siente por mí.

$\mathrm{Ap}+$ 41.- Siento que realmente me valora.

$\mathrm{E}+$ 42.- El (ella) capta exactamente la forma en que yo siento las cosas que experimento.

Ia - 43.- Aprueba algunas cosas que hago y no aprueba abiertamente otras.

$\mathrm{C}+$ 44.- Está dispuesto(a) a expresarme lo que realmente está pensando, incluyendo cualquier sentimiento hacia si mismo(a) o hacia mí.

Ap - 45.- No le gusto por lo que soy.

E- 46.- A veces, él (ella) cree que mis sentimien- tos por algo en particular son mucho más fuertes que como yo los siento en realidad.

Ia + 47.- El que yo esté de buen ánimo o de mal genio no le hace sentir ni más ni menos aprecio por mí.

$\mathrm{C}+$ 48.- En nuestra relación se muestra tal como es.

Ap - 49.- Parece que le molesto e irrito.

E- $\quad$ 50.- No percibe cuán sensible soy respectoa algunas de las cosas que conversamos.

$\mathrm{Ia}+\quad$ 51.- El que las ideas o sentimientos que yo exprese sean "buenos" o "malos" no parece influír en sus sentimientos hacia mí.

C - 52.- A veces siento que lo que muestra externamente es muy diferente de lo que realmente siente.

Ap - 53.- A veces me desprecia.

$\mathrm{E}+$ 54.- Me entiende.

Ia - 55.- Hay ocasiones en que soy más valioso para él (ella) que en otras.

$\mathrm{C}+\quad$ 56.- Siento que él (ella) no ha tratado de ignorar nada de lo que siente hacia mí.

$\mathrm{Ap}+$ 57.- Se interesa realmente en mí.

E- 58.- Su conducta conmigo es generalmente tan fija y automática que no consigo llegar a él (ella).

Ia + 59.- Creo que nada que yo diga o haga realmente cambie lo que él (ella) siente por mí.

C - $\quad$ 60.- Con frecuencia, lo que me dice da una impresión equivocada de todo lo que piensa o siente en ese momento.

$A p+$ 61.- Siente un cariño profundo por mí.

$\mathrm{E}+$ 62.- Cuando yo me siento dolido o alterado, él (ella) puede reconocer y distinguir claramente mis sentimientos, sin alterarse él (ella).

Ia - 63.- Lo que otros piensan de míafecta (o afectaria, si lo supiera) lo que siente por mí.

C - 64.- Creo que algunos de sus sentimientos, de los cuales no me habla, están produciendo dificultades en nuestra relación. 
HOJADERESPUESTAS

INVENTARIO DE LA RELACION

G.T. BARRETT-LENNARD

\begin{tabular}{|c|c|c|c|}
\hline 1 & _ 17 & 33 & 49 \\
\hline 2 & 18 & 34 & 50 \\
\hline 3 & 19 & 35 & 51 \\
\hline 4 & 20 & 36 & 52 \\
\hline 5 & 21 & 37 & 53 \\
\hline 6 & 22 & 38 & 54 \\
\hline 7 & 23 & 39 & 55 \\
\hline 8 & -24 & 40 & 56 \\
\hline 9 & 25 & 41 & 57 \\
\hline 10 & 26 & 42 & 58 \\
\hline 11 & 27 & 43 & 59 \\
\hline 12 & 28 & 44 & 60 \\
\hline 13 & 29 & 45 & 61 \\
\hline 14 & 30 & 46 & 62 \\
\hline 15 & 31 & 47 & 63 \\
\hline 16 & -32 & 48 & 64 \\
\hline
\end{tabular}

Nombre de quien responde el Inventario:

Persona Evaluada:

Fecha de Hoy:

Puntajes:

E:

C:

Ap:

Ia:

\section{CORRECCIÓN}

Se sigue el procedimiento ya señalado anteriormente. La Hoja de Respuestas -diseñada para este artículo- intenta facilitar la corrección. Al confeccionar cuatro plantillas -una para cada Escala-, se deberán hacer "ventanillas" en cada una de ellas, como se indica a continuación:

Para la plantilla de la Escala de Aprecio, en cuatro lineas horizontales: ítems

$$
\begin{array}{ll}
\text { 1-17-33-49; } & \text { 5-21-37-53; } \\
\text { 9-25-41-57; } & \text { y13-29-45-61. }
\end{array}
$$

Para la plantilla de la Escala de Empatía, las siguientes cuatro lineas:

$$
\begin{aligned}
2-18-34-50 ; & 6-22-38-54 ; \\
10-26-42-58 ; & \text { y } 14-30-46-62 .
\end{aligned}
$$

Para plantilla de la Escala de Incondicionalidad del Aprecio, las siguientes cuatro:

$$
\begin{array}{cl}
\text { 3-19-35-51; } & \text { 7-23-39-55; } \\
11-27-43-59 ; & \text { y15-31-47-63. }
\end{array}
$$

Por último, para la plantilla de la Escala de Congruencia, las siguientes cuatro:

$$
\begin{aligned}
\text { 4-20-36-52; } & 8-24-40-56 ; \\
12-28-44-60 ; & \text { y16-32-48-64. }
\end{aligned}
$$

